\title{
Geometrical Conditions for Output Depending Observability Normal Form
}

\author{
G. Zheng, D. Boutat and J-P. Barbot
}

\begin{abstract}
We give geometrical conditions, which guarantee the existence of a diffeomorphism in order to transform a nonlinear system without inputs into a canonical normal form depending on its output. Moreover we extend our results to a class of systems with inputs. We end this paper by some examples and its simulations to highlight the proposed algo rithm.
\end{abstract}

Index Terms-Algebraic/geometric methods, Nonlinear systems, output feedback and observers.

\section{INTRODUCTION}

In order to use the Luenberger's observer [9] for nonlinear systems, the so-called problem of the Nonlinear Observer Canonical Form (NOCF) was born. In [12] authors give sufficient and necessary conditions which guarantee the existence of a diffeomorphism and of an output injection to transform a single output nonlinear system without inputs into NOCF form. For a multi-output nonlinear system without inputs, it was solved in [13] and [16]. Another approach was introduced for the analytical systems in [11] by assuming that the spectrum of the linear part must lie in the Poincare domain and it was generalized in [14] by assuming that the spectrum of the linear part must lie in the Siegel domain. Other approaches using quadratic normal forms were given in [1] and [3]. Moreover, [10] gave the sufficient and necessary geometrical conditions to transform a nonlinear system into a so-called output-dependent time scaling linear canonical form, while [5] gave the dual geometrical conditions of [10].

In this paper, we study the geometrical conditions to guarantee the existence of a local diffeomorphism $z=\phi(x)$ which transforms the locally observable dynamical system into a NOCF form depending on the output [17]. More precisely, let us consider the following system:

$$
\left\{\begin{array}{l}
\dot{x}=f(x), \\
y=h(x),
\end{array}\right.
$$

where $x \in U \subset \mathbb{R}^{n}$ and $h: U \subset \mathbb{R}^{n} \rightarrow \mathbb{R}$ are sufficiently smooth. We are interested in deducing necessary and sufficient geometrical conditions to guarantee the existence of a local diffeomorphism $z=\phi(x)$ which transforms system (1) into the following form

$$
\left\{\begin{array}{l}
\dot{z}=A(y) z+\beta(y) \\
y=z_{n}=C z
\end{array}\right.
$$

G. Zheng is with LJK, UMR CNRS 5224, 51 rue des Mathmatiques, 38041 Grenoble, France gang. zheng@imag. fr

D. Boutat is with LVR ENSI, 10 Boulevard de Lahitolle, 18020 Bourges, France driss.boutateensi-bourges.fr

J-P. Barbot is with ECS ENSEA, 6 Avenue du Ponceau, 95014 Cergy-Pontoise, and Project ALIEN, INRIA-Futur, France barbotensea.fr where

$$
A(y)=\left(\begin{array}{cccc}
0 & \cdots & 0 & 0 \\
\alpha_{1}(y) & \cdots & 0 & 0 \\
\vdots & \ddots & \ldots & \vdots \\
0 & \cdots & \alpha_{n-1}(y) & 0
\end{array}\right), \beta(y)=\left(\begin{array}{c}
\beta_{1}(y) \\
\beta_{2}(y) \\
\vdots \\
\beta_{n}(y)
\end{array}\right) \text {, }
$$

and $\alpha_{i}(y) \neq 0$ for $\left.y \in\right]-a, a[$ and $a>0$. This kind of linearization is called Single Output Dependent Observability normal form (SODO normal form).

For dynamical systems in the form (2), some types of high-gain observer [6], [4], [7] can be synthesized. Here we may apply the following high gain observer [2]:

$$
\left\{\begin{array}{l}
\dot{\hat{z}}=A(y) \hat{z}+\beta(y)-\Gamma^{-1}(y) R_{\rho}^{-1} C^{T}(C \hat{z}-y), \\
0=-\rho R_{\rho}-\bar{A}^{T} R_{\rho}-R_{\rho} \bar{A}+C^{T} C .
\end{array}\right.
$$

where $\Gamma(y)$ is the $n \times n$ diagonal matrix $\Gamma(y)=$ $\operatorname{diag}\left[\prod_{i=1}^{n-1} \alpha_{i}(y), \prod_{i=2}^{n-1} \alpha_{i}(y), \cdots, \alpha_{n-1}(y), 1\right]$ and $\bar{A}$ is the $n \times n$ matrix defined as follows

$$
\bar{A}=\left(\begin{array}{cccc}
0 & \cdots & 0 & 0 \\
1 & \cdots & 0 & 0 \\
\vdots & \ddots & \vdots & \vdots \\
0 & \cdots & 1 & 0
\end{array}\right)
$$

Indeed, here the output of system (2) is considered as an input of (3). Setting $e=z-\hat{z}$, the observation error can be obtained as follows:

$$
\dot{e}=\left(A(y)-\Gamma^{-1}(y) R_{\rho}^{-1} C^{T} C\right) e .
$$

And the convergence of such observer is proved in [2], thus in section 4 we simply highlight the design of such observer for systems in the form (2).

This paper is organized as follows. The next section addresses basic notations. In section 3, we present our main theorem to guarantee the studied transform for nonlinear system without inputs. Section 4 is devoted to the generalization of our results to a class of systems with inputs, and some practical particular cases are studied in the same section.

\section{NOTATIONS AND TECHNICAL RESULTS}

Throughout this article, $L_{f}^{i-1} h$ for $1 \leq i \leq n$ denotes the $(i-1)^{t h}$ Lie derivative of output $h$ in the direction of $f$, and set $\theta_{i}=d L_{f}^{i-1} h$ as its differential. Assume that system (1) is locally observable, thus $\theta=\left(\theta_{1}, \cdots, \theta_{n}\right)^{T}$ is 
a basis of the cotangent bundle $T^{*} U$ of $U$. Then, we also consider the vector field $\tau_{1}$ defined in [12] as follows

$$
\left\{\begin{array}{l}
\theta_{i}\left(\tau_{1}\right)=0, \text { for } 1 \leq i \leq n-1, \\
\theta_{n}\left(\tau_{1}\right)=1,
\end{array}\right.
$$

and by induction we define

$$
\tau_{k}=(-1)^{k-1} a d_{f}^{k-1}\left(\tau_{1}\right), \text { for } 2 \leq k \leq n .
$$

It is clear that $\left\{\tau_{1}, \cdots, \tau_{n}\right\}$ is a basis of the tangent bundle $T U$ of $U$.

Let us recall a famous result from [12].

Theorem 1: The following conditions are equivalent

i) There exist a diffeomorphism and an output injection which transform system (1) into normal form (2) with $\alpha_{k}(y)=1$ for $1 \leq k \leq n-1$.

ii) $\left[\tau_{i}, \tau_{j}\right]=0$ for $1 \leq i, j \leq n$.

If for some $1 \leq k \leq n-1$ the functions $\alpha_{k}(y)$ in the form (2) are not constant, then $i i$ ) of Theorem 1 is not fulfilled. In [10], authors considered the case of $\alpha_{i}(y)=s(y)$ for $1 \leq i \leq n-1$. Firstly, for $j \geq 2$, they defined

$$
l_{j}=\frac{j(j-1)}{2}+1
$$

Then, for the non-trivial case $n \geq 2$, according to the following equality:

$$
d\left(\theta_{n}\left(\tau_{2}\right)\right)=l_{n} \lambda \theta_{2} \text { modspan }\left\{\theta_{1}\right\}
$$

we can uniquely determine the smooth function $\lambda$. In the following, we calculate $s(y)$ as follows:

$$
s=\exp \sigma
$$

where $\sigma$ is a solution of the following equation

$$
L_{\tau_{j+1}} \sigma= \begin{cases}0 & \text { if } 0 \leq j \leq n-2 \\ \lambda & \text { if } j=n-1\end{cases}
$$

By defining

$$
\left\{\begin{array}{l}
\widetilde{\tau}_{1}=s^{n-1} \tau_{1} \\
\widetilde{\tau}_{i+1}=\left[\widetilde{\tau}_{i}, \frac{f}{s}\right]
\end{array}\right.
$$

for $1 \leq i \leq n-1$, then Theorem 2 in [10] can be stated as follows:

Theorem 2: The following conditions are equivalent

i) There exist a diffeomorphism and an output injection which transform system (1) into normal form (2) with $\alpha_{k}(y)=s(y)$ for $1 \leq k \leq n-1$.

ii) $\left[\widetilde{\tau}_{i}, \widetilde{\tau}_{j}\right]=0$ for $1 \leq i, j \leq n$.

This paper is for the purpose of studying the case where $\alpha_{i}(y)$ for $1 \leq i \leq n-1$ is neither constant, nor scalar function of the output, i.e. $\alpha_{i}(y)$ can be not equal to $\alpha_{j}(y)$ for $1 \leq i, j \leq n-1$ and $i \neq j$. The main result is given in the next section by assuming that a family of functions $\alpha_{i}(y)$ for $1 \leq i \leq n-1$ can be uniquely determined. The details on calculation of such family of functions is exhaustively discussed in [18].

\section{MAIN RESUlT}

If there exists a diffeomorphism which transforms system (1) into form (2), then Proposition 2.3 of [18] gives all $\alpha_{i}$ for $1 \leq i \leq n-1$. Therefore, let us consider a new family of vector fields defined as follows:

$$
\widetilde{\tau}_{1}=\prod_{i=1}^{n} \alpha_{i}(y) \tau_{1} \text { and } \widetilde{\tau}_{i+1}=\frac{1}{\alpha_{i}}\left[\widetilde{\tau}_{i}, f\right]
$$

for $1 \leq i \leq n-1$. Set

$$
\theta\left(\widetilde{\tau}_{1}, \cdots, \widetilde{\tau}_{n}\right)=\left(\begin{array}{ccccc}
0 & 0 & \cdots & 0 & 1 \\
0 & \vdots & \cdots & \pi_{n-1} & \widetilde{l}_{2, n} \\
\vdots & \ldots & \ddots & \cdots & \vdots \\
\vdots & \pi_{2} & \cdots & \cdots & \vdots \\
\pi_{1} & \widetilde{l}_{n, 2} & \cdots & \cdots & \widetilde{l}_{n, n}
\end{array}\right):=\widetilde{\Lambda},
$$

where

$$
\widetilde{l}_{k, j}=\theta_{k}\left(\widetilde{\tau}_{j}\right) \text { for } 2 \leq k \leq n \text { and } n-k+2 \leq j \leq n .
$$

Consider the following $R^{n}$-valued form $\omega$

$$
\omega=\widetilde{\Lambda}^{-1} \theta:=\left(\omega_{1}, \omega_{2}, \cdots, \omega_{n}\right)^{T},
$$

where, for $1 \leq s \leq n$, we have

$$
\omega_{s}=\sum_{m=1}^{n} r_{s, m} \theta_{m}
$$

Then, the following algorithm gives all the components of $\omega$.

Algorithm 1:

$$
\begin{aligned}
& \text { for } 1 \leq j \leq n, \\
& \qquad r_{n, j}=\cdots=r_{n-j+2, j}=0 \text { and } r_{n-j+1, j}=1 . \\
& \text { for } 2 \leq k \leq n-1 \text { and } 1 \leq j \leq n, \\
& \qquad r_{n-k, j}=-\sum_{i=2}^{k} \widetilde{l}_{k, n-k+i-(j-1)} r_{n-k+i-(j-1), j},
\end{aligned}
$$

and then, equation (8) becomes: $\omega_{s}=\sum_{m=1}^{n-s+1} r_{s, m} \theta_{m}$.

Theorem 3: The following conditions are equivalent

1) There exists a diffeomorphism which transforms system (1) into a SODO normal form (2).

2) There exists a family of functions $\alpha_{i}(y)$ for $1 \leq i \leq$ $n-1$ such that the family of vector fields $\widetilde{\tau}_{i}$ for $1 \leq$ $i \leq n$ defined in (6) satisfies the following commutativity conditions

$$
\left[\widetilde{\tau}_{i}, \widetilde{\tau}_{j}\right]=0, \text { for } 1 \leq i, j \leq n .
$$

3) There exists a family of functions $\alpha_{i}(y)$ for $1 \leq i \leq$ $n-1$ such that the $R^{n}$-valued form $\omega$ defined in (7) satisfies the following condition

$$
d \omega=0 .
$$

Proof: Assume that there exists a diffeomorphism which transforms system (1) into form (2), then we compute 
$\alpha_{i}(y)$ for $1 \leq i \leq n-1$ according to Proposition 2.3 in [18]. Thus, it is easy to show that $\tau_{1}=\frac{1}{\pi_{1}} \frac{\partial}{\partial z_{1}}$ which yields that $\widetilde{\tau}_{1}=\frac{\partial}{\partial z_{1}}$ and then, by construction we obtain $\widetilde{\tau}_{i}=\frac{\partial}{\partial z_{i}}$ for $2 \leq i \leq n$. Consequently, we have $\left[\widetilde{\tau}_{i}, \widetilde{\tau}_{j}\right]=0$ for $1 \leq i, j \leq n$.

Reciprocally, assume that there exist $\alpha_{i}>0$ for $1 \leq i \leq$ $n-1$ such that $\left[\widetilde{\tau}_{i}, \widetilde{\tau}_{j}\right]=0$ for $1 \leq i, j \leq n$, then it is wellknown ([8], [15]) that we can find a local diffeomorphism $\phi=z$ such that

$$
\phi_{*}\left(\widetilde{\tau}_{i}\right)=\frac{\partial}{\partial z_{i}} .
$$

As $\phi_{*}\left(\tilde{\tau}_{i}\right)=\frac{\partial}{\partial z_{i}}$ is constant, hence

$$
\frac{\partial}{\partial z_{i}} \phi_{*}(f)=\phi_{*}\left(\left[\widetilde{\tau}_{i}, f\right]\right)=\alpha_{i} \phi_{*}\left(\widetilde{\tau}_{i+1}\right)=\alpha_{i} \frac{\partial}{\partial z_{i+1}},
$$

thus $\frac{\partial}{\partial z_{i}} \phi_{*}(f)=\alpha_{i} \frac{\partial}{\partial z_{i+1}}$ for $1 \leq i \leq n-1$. Consequently, by integration we obtain: $\phi_{*}(f)=A(y) z+\beta(y)$.

Moreover, as $d h \circ \widetilde{\tau}_{i}=0$ for $1 \leq i \leq n-1$ and $d h \circ \widetilde{\tau}_{n}=1$, we obtain $h \circ \phi^{-1}=z_{n}$.

Finally, in order to prove that in Theorem (3) Condition 2 ) is equivalent to Condition 3), it is sufficient to prove that equation (9) is equivalent to equation (10).

Recall that for any two vector fields $X, Y$, we have

$$
d \omega(X, Y)=L_{X}(\omega(Y))-L_{Y}(\omega(X))-\omega([X, Y]) .
$$

Setting $X=\widetilde{\tau}_{i}$ and $Y=\widetilde{\tau}_{j}$, we obtain

$$
d \omega\left(\widetilde{\tau}_{i}, \widetilde{\tau}_{j}\right)=L_{\widetilde{\tau}_{i}} \omega\left(\widetilde{\tau}_{j}\right)-L_{\widetilde{\tau}_{j}} \omega\left(\widetilde{\tau}_{i}\right)-\omega\left(\left[\widetilde{\tau}_{i}, \widetilde{\tau}_{j}\right]\right) .
$$

As $\omega\left(\widetilde{\tau}_{j}\right)$ and $\omega\left(\widetilde{\tau}_{i}\right)$ are constant, then we have

$$
d \omega\left(\widetilde{\tau}_{i}, \widetilde{\tau}_{j}\right)=-\omega\left(\left[\widetilde{\tau}_{i}, \widetilde{\tau}_{j}\right]\right)
$$

Because $\omega$ is an isomorphism and $\left(\widetilde{\tau}_{i}\right)_{1 \leq i \leq n}$ is a basis of $T U$, then equation (9) is equivalent to equation (10).

Remarks 1: i) The $R^{n}$-valued form $\omega$ can be viewed as an isomorphism $T U^{n} \rightarrow U \times \mathbb{R}^{n}$ which brings each $\widetilde{\tau}_{i}$ to the canonical vector basis $\frac{\partial}{\partial z_{I}}$. Moreover, $d \omega=0$ means that there is a local diffeomorphism $\phi: U \rightarrow U$ such that $\omega$ is the tangent map of $\phi$.

ii) The diffeomorphism $\phi(x)=z$ is determined by $\omega=$ $\phi_{*}(x)$, which can be given locally as follows

$$
z_{i}=\phi_{i}(x)=\int_{\gamma} \omega_{i}+\phi_{i}(0) \text { for } 1 \leq i \leq n,
$$

where $\gamma$ is a smooth path from 0 to $x$ lying in a neighborhood $V_{0} \subseteq U$ of 0 .

The following simple example is studied in order to illustrate Theorem 3.

Example 1: Let us consider the following system

$$
\left\{\begin{array}{l}
\dot{x}_{1}=\frac{\gamma(y)}{1+x_{4}} x_{1} x_{3} \\
\dot{x}_{2}=\frac{\beta(y)}{1+x_{4}} x_{1} \\
\dot{x}_{3}=\mu(y) x_{2} \\
\dot{x}_{4}=\gamma(y) x_{3} \\
y=x_{4}
\end{array}\right.
$$

which gives

$$
\left\{\begin{aligned}
\theta_{1}= & d x_{4} \\
\theta_{2}= & \gamma d x_{3}+\gamma^{\prime} x_{3} d x_{4} \\
\theta_{3}= & \gamma \mu d x_{2}+2 \gamma^{\prime} \gamma x_{3} d x_{3}+\left((\gamma \mu)^{\prime} x_{2}+\left(\gamma^{\prime} \gamma\right)^{\prime} x_{3}^{2}\right) d x_{4} \\
\theta_{4}= & \gamma \mu \frac{\beta}{1+x_{4}} d x_{1}+\left(2 \gamma^{\prime} \mu+(\gamma \mu)^{\prime}\right) \gamma x_{3} d x_{2} \\
& +\left(2 \gamma^{\prime} \gamma \mu x_{2}+\gamma(\gamma \mu)^{\prime} x_{2}+3 \gamma\left(\gamma^{\prime} \gamma\right)^{\prime} x_{3}^{2}\right) d x_{3} \\
& +O^{[2]}\left(x_{1}, x_{2}, x_{3}\right) \theta_{1}
\end{aligned}\right.
$$

Then we have $\tau_{1}=\frac{1+x_{4}}{\gamma \mu \beta} \frac{\partial}{\partial x_{1}}$.

According to Proposition 2.3 in [18], $\alpha_{1}=\frac{c_{1}}{c_{2}} \beta, \alpha_{2}=\frac{c_{2}}{c_{3}} \mu$ and $\alpha_{3}=c_{3} \gamma$, so the new vector fields are

$$
\begin{aligned}
& \widetilde{\tau}_{1}=c_{1}\left(1+x_{4}\right) \frac{\partial}{\partial x_{1}}, \widetilde{\tau}_{2}=c_{2} \frac{\partial}{\partial x_{2}}, \\
& \widetilde{\tau}_{3}=c_{3} \frac{\partial}{\partial x_{3}}, \widetilde{\tau}_{4}=\frac{\partial}{\partial x_{4}}+\frac{x_{1}}{1+x_{4}} \frac{\partial}{\partial x_{1}} .
\end{aligned}
$$

It is clear that $\left[\widetilde{\tau}_{i}, \widetilde{\tau}_{j}\right]=0$ for all $1 \leq i, j \leq 4$. Therefore, according to Theorem 3 , system (11) can be transformed into SODO normal form (2).

Moreover as $\widetilde{\Lambda}=\theta\left(\widetilde{\tau}_{1}, \cdots, \widetilde{\tau}_{n}\right)$, a straightforward computation gives

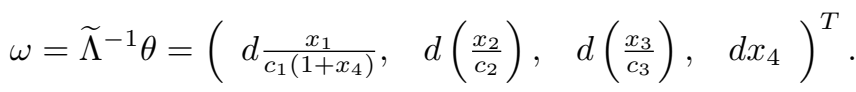

As $\omega=d \phi$, thus the diffeomorphism which transforms system (11) into SODO normal form (2) is

$$
\phi(x)=z=\left(\frac{x_{1}}{c_{1}\left(1+x_{4}\right)}, \frac{x_{2}}{c_{2}}, \frac{x_{3}}{c_{3}}, x_{4}\right)^{T} .
$$

with which system (11) could be transformed into

$$
\left\{\begin{array}{l}
\dot{z}_{1}=0, \\
\dot{z}_{2}=\frac{c_{1}}{c_{2}} \beta(y) z_{1}, \\
\dot{z}_{3}=\frac{c_{2}}{c_{3}} \mu(y) z_{2}, \\
\dot{z}_{4}=c_{3} \gamma(y) z_{3} .
\end{array}\right.
$$

So far, in this paper, we have only considered systems without inputs. The next section is devoted to systems that are also driven by an input term.

\section{EXTENSION TO SYSTEMS WITH INPUTS}

Consider a system with inputs in the following form

$$
\left\{\begin{array}{l}
\dot{x}=f(x)+g(x, u) \\
y=h(x)
\end{array}\right.
$$

where $x \in U \subset \mathbb{R}^{n}, f: U \subset \mathbb{R}^{n} \rightarrow \mathbb{R}^{n}, g: U \times \mathbb{R}^{m} \rightarrow \mathbb{R}^{n}$, $h: U \subset \mathbb{R}^{n} \rightarrow \mathbb{R}$ are analytic functions and for $x \in U$, $g(x, 0)=0$.

For system (12), the SODO normal form along its output trajectory $y(t)$ is as follows

$$
\left\{\begin{array}{l}
\dot{z}=A(y) z+\beta(y)+\eta(y, u) \\
y=z_{n}=C z
\end{array}\right.
$$

where $A(y)$ and $\beta(y)$ are given in (2) and $\eta(y, u)=$ $\left[\eta_{1}(y, u), \quad \eta_{2}(y, u), \cdots, \quad \eta_{n}(y, u)\right]^{T}$.

Theorem 4: System (12) can be transformed into SODO normal form (13) by a diffeomorphism if and only if

$i$ ) one of conditions in Theorem 3 is fulfilled. 
ii) $\left[g, \widetilde{\tau}_{i}\right]=0$ for $1 \leq i \leq n-1$.

Proof: From Theorem 3, we can state that there exists a diffeomorphism $\phi$ such that

$$
\phi_{*}(f)=A(y) z+\beta(y) .
$$

For $1 \leq i \leq n-1$, because $\phi_{*}\left(\widetilde{\tau}_{i}\right)=\frac{\partial}{\partial z_{i}}$ is constant, hence we have

$$
\frac{\partial}{\partial z_{i}} \phi_{*}(g)=\phi_{*}\left(\left[g, \widetilde{\tau}_{i}\right]\right)=0 .
$$

Therefore $\phi_{*}(g)=\eta(y, u)$. Thus, we obtain the form (13).

Remark 1: If $g(x, u)=g_{1}(x) u_{1}+\cdots+g_{m}(x) u_{m}$, and also both conditions $i$ ) and $i$ ) of Theorem 4 are fulfilled, then

$$
\eta(y, u)=B_{1}(y) u_{1}+\cdots+B_{m}(y) u_{m} .
$$

Let us now study some special cases of the term $\eta(y, u)$.

Corollary 1: Assume that conditions $i$ ) and $i i$ ) of Theorem 4 are fulfilled,

a) if $\left[g, \widetilde{\tau}_{n}\right]=0$, then

$$
\eta(y, u)=\eta(u) .
$$

b) if $g(x, u)=g_{1}(x) u_{1}+\cdots+g_{m}(x) u_{m}$ and

$$
\left[g_{k}, \widetilde{\tau}_{i}\right]=0, \text { for } 1 \leq i \leq n \text { and } 1 \leq k \leq m,
$$

then

$$
\eta(y, u)=B_{1} u_{1}+\cdots+B_{m} u_{m}
$$

where $B_{i}$ are constant vector fields.

Example 2: Let us consider the following system

$$
\left\{\begin{array}{l}
\dot{x}_{1}=\frac{\gamma(y)}{1+x_{3}} x_{1} x_{2}+\frac{x_{1}}{1+x_{3}} u \\
\dot{x}_{2}=\frac{\mu(y)}{1+x_{3}} x_{1} \\
\dot{x}_{3}=\gamma(y) x_{2}+u \\
y=x_{3}
\end{array}\right.
$$

A similar computation gives: $\alpha_{1}(y)=\frac{c_{1}}{c_{2}} \mu(y)$ and $\alpha_{2}(y)=$ $c_{2} \gamma(y)$. Therefore, we obtain $\widetilde{\tau}_{1}=c_{1}\left(1+x_{3}\right) \frac{\partial}{\partial x_{1}}, \widetilde{\tau}_{2}=$ $c_{2} \frac{\partial}{\partial x_{2}}$ and $\widetilde{\tau}_{3}=\frac{\partial}{\partial x_{3}}+\frac{x_{1}}{1+x_{3}} \frac{\partial}{\partial x_{1}}$.

As $g=\frac{x_{1}}{1+x_{3}} \frac{\partial}{\partial x_{1}}+\frac{\partial}{\partial x_{3}}=\widetilde{\tau}_{3}$ then $\left[g, \widetilde{\tau}_{1}\right]=\left[g, \widetilde{\tau}_{2}\right]=0$ and system (14) is transformed into

$$
\left\{\begin{array}{l}
\dot{z}_{1}=0 \\
\dot{z}_{2}=\frac{c_{1}}{c_{2}} \mu(y) z_{1} \\
\dot{z}_{3}=c_{2} \gamma(y) z_{2}+u \\
y=z_{3}
\end{array}\right.
$$

by the following diffeomorphism

$$
\phi(x)=z=\left(\frac{x_{1}}{c_{1}\left(1+x_{3}\right)}, \frac{x_{2}}{c_{2}}, x_{3}\right)^{T} .
$$

Following the proposed high gain observer in the form (3), the corresponding observer for the system (15) can be designed as follows:

$$
\left\{\begin{array}{l}
\dot{\hat{z}}_{1}=-\frac{\rho^{3}}{\gamma \mu}\left(\hat{z}_{3}-z_{3}\right) \\
\dot{\hat{z}}_{2}=\frac{c_{1}}{c_{2}} \mu(y) \hat{z}_{1}-3 \frac{\rho^{2}}{\gamma}\left(\hat{z}_{3}-z_{3}\right) \\
\dot{\hat{z}}_{3}=c_{2} \gamma(y) \hat{z}_{2}-3 \rho\left(\hat{z}_{3}-z_{3}\right)+u
\end{array}\right.
$$

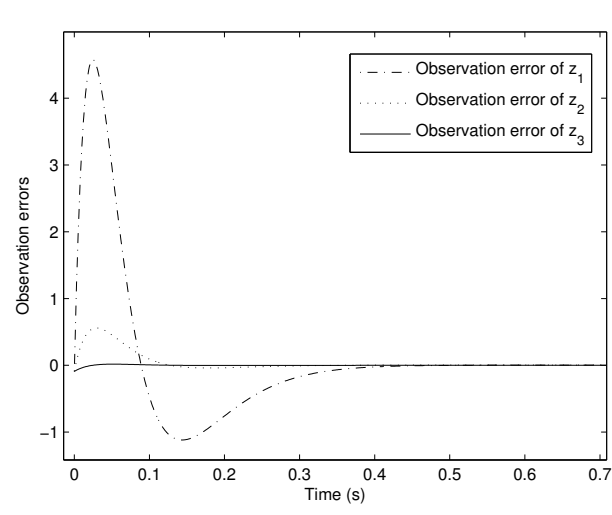

Fig. 1. Observation errors of $z_{1}, z_{2}$ and $z_{3}$

where $\rho$ is the tunable gain. For a more specific but simple simulation, choose $c_{1}=c_{2}=1, u(t)=1, \mu(y)=1+y^{2}$, and $\gamma(y)=2+\cos (y)$. Its simulation results are presented in Fig. 1 which presents the convergence of system's states and their estimations.

In addition, in order to solve the left invertibility problem, the Observability Matching Condition (OMC) for system (12) with $m=1$ is as follows

$$
\left\{\begin{array}{l}
L_{g} L_{f}^{i-1} h=0, \forall x \in U, 1 \leq i \leq n-1, \\
L_{g} L_{f}^{n-1} h \neq 0 .
\end{array}\right.
$$

Corollary 2: Assume conditions $i$ ) and $i i$ ) of Theorem 4 are fulfilled and the OMC is verified then

$$
\eta(y, u)=\left[\begin{array}{llll}
\eta_{1}(y, u), & 0, & \cdots, & 0
\end{array}\right]^{T} .
$$

Remark 2: The OMC for system (12) with $m=1$ is equivalent to $g \in \operatorname{span}\left\{\widetilde{\tau}_{1}\right\}$. 2.

We give another example in order to highlight Corollary

Example 3: Consider the following system

$$
\left\{\begin{array}{l}
\dot{x}_{1}=u \\
\dot{x}_{2}=\mu(y) x_{1}+\mu(y) x_{1}^{2}+\frac{x_{2}}{1+x_{1}} u, \\
\dot{x}_{3}=\gamma(y) \frac{x_{2}}{1+x_{1}} \\
y=x_{3}
\end{array}\right.
$$

A straightforward computation gives $\alpha_{1}(y)=\frac{c_{1}}{c_{2}} \mu(y)$ and $\alpha_{2}(y)=c_{2} \gamma(y)$. Thus, we have $\widetilde{\tau}_{1}=c_{1} \frac{\partial}{\partial x_{1}}+c_{1} \frac{x_{2}}{1+x_{1}} \frac{\partial}{\partial x_{2}}$, $\widetilde{\tau}_{2}=c_{2}\left(1+x_{1}\right) \frac{\partial}{\partial x_{2}}$ and $\widetilde{\tau}_{3}=\frac{\partial}{\partial x_{3}}$.

As $g \in \operatorname{span}\left\{\widetilde{\tau}_{1}\right\}$, then the OMC condition is fulfilled, therefore system (16) could be transformed by the diffeomorphism: $\phi(x)=z=\left(\frac{x_{1}}{c_{1}}, \frac{x_{2}}{c_{2}\left(1+x_{1}\right)}, x_{3}\right)^{T}$ into

$$
\left\{\begin{array}{l}
\dot{z}_{1}=\frac{u}{c_{1}} \\
\dot{z}_{2}=\frac{c_{1}}{c_{2}} \mu(y) z_{1}, \\
\dot{z}_{3}=c_{2} \gamma(y) z_{2}, \\
y=z_{3}
\end{array}\right.
$$


And it is obvious that the input $u$ can be represented as an algebraic function of the output and its derivative as follows:

$$
\begin{gathered}
u=\frac{\gamma^{2} \mu \dddot{y}-\gamma \mu \gamma^{\prime} \ddot{y}-\gamma^{2} \mu^{\prime} \ddot{y}-\gamma \mu \gamma^{\prime \prime} \dot{y}+\gamma \mu\left(\gamma^{\prime}\right)^{2} \dot{y}+\gamma \gamma^{\prime} \mu^{\prime} \dot{y}}{\gamma^{3} \mu^{2}} \\
\text { V. CONCLUSION }
\end{gathered}
$$

This paper studied the SODO normal form. Geometrical conditions, which allow us to determine whether a nonlinear system can be transformed locally into the studied normal form by means of a diffeomorphism and of an output injection, are deduced. Moreover, an extension of our results is stated for a class of nonlinear systems with inputs.

\section{REFERENCES}

[1] L. Boutat-Baddas, D. Boutat, J-P. BARbot And R. TAUleigne, Quadratic observability normal form, in Proc. of IEEE CDC (2001).

[2] K. Busawon, M. Farza AND H. HAMmouri, A simple observer for a class of nonlinear systems, Appl. Math. Lett, Vol. 11, No.3, (1998) pp. 27-31.

[3] S. Chabraoui, D. Boutat, L.Boutat-Baddas and J.P. Barbot, Observability quadratic characteristic numbers, in Proc. of IEEE CDC (2001).

[4] J. P. Gauthier and I. KUPKa, Deterministic observation theory and applications, Cambridge University Press, 2001.

[5] M. GuAY, Observer linearization by output diffeomorphism and output-dependent time-scale transformations, NOLCOS'01 Saint Petersburg, Russia ,(2001) pp.1443-1446.

[6] H. Hammouri AND J.P. GauthiER, Bilinearization up to the output injection, System and Control Letters, 11 , (1988) pp.139-149.

[7] H. Hammouri AND J. Morales, Observer synthesis for state-affine systems, in Proc. of IEEE CDC (1990).

[8] A. ISIDORI, Nonlinear control systems, 2nd edtion, Berlin: SpringerVerlag,(1989).

[9] D. Luenberger, An introduction to observers, in IEEE Transactions on Automatic Control 16 (6), (1971) pp. 596-602.

[10] W. Respondek, A. Pogromsky And H. NiJmeijer, Time scaling for observer design with linearization error dynamics, in IEEE Transactions on Automatic Control 3, (1989) pp. 199-216.

[11] N. KAZANTZIS AND C. KRAVARIS, Nonlinear observer design using Lyapunov's auxiliary theorem, in Systems \& Control Letters, Vo 34, (1998) pp. 241-247.

[12] A. KRENER AND A. ISIDORI, Linearization by output injection and nonlinear observer, in Systems \& Control Letters, Vo 3, (1983) pp.4752.

[13] A. KRENER AND W. ResPondeK, Nonlinear observer with linearizable error dynamics, in SIAM J. Control and Optimization, Vol 30, No 6, (1985) pp.197-216.

[14] A. Krener and M. Q. Xiao, Nonlinear observer design in the Siegel domain through coordinate changes, in Proc of the 5th IFAC Symposium, NOLCOS01, Saint-Petersburg, Russia, (2001) pp.557562.

[15] H. NiJmeijer and A.J. VAn Der Schaft, Nonlinear dynamical control systems, Springer (1996).

[16] X.H. XIA AND W.B. GAO, Nonlinear observer design by observer error linearization, in SIAM J. Control and Optimization, Vol 27,(1989) pp. 199-216.

[17] G. Zheng, D. Boutat And J.P. Barbot, Output dependent observability linear normal form, in Proc. of IEEE CDC (2005).

[18] G. Zheng, D. Boutat And J.P. Barbot, Single output-dependent observability normal form, in SIAM J. Control and Optimization, Vol 46, (2007) pp. 2242-2255. 